\title{
CHARACTER INDUCTION IN P-GROUPS
}

\author{
TERESA L. SANTA COLOMA \\ Department of Mathematical Sciences \\ Loyola University \\ New Orleans, LA 70118 \\ (Received September 16, 1985 and in revised form October 3, 1985)
}

ABSTRACT. Let $G$ be a finite $p$-group and let $\chi$ be an irreducible character of $G$. Then $X$ is monomial; that is, $X=\lambda^{G}$, where $\lambda$ is a linear character of some subgroup of $G$. We are interested in locating subgroups of $G$ which induce the character $X$.

KEYWORDS AND PHRASES. induced character, support group, inertia group. 1980 Mathematics Subject Classification: $20 \mathrm{c} 15,20 \mathrm{c} 30$.

\section{INTRODUCTION}

For $G$ a finite $p$-group and $X \in \operatorname{Irr}(G)$ (the irreducible characters of $G$ ), $X$ non-linear $(X(1)=1)$ it is known that there is some subgroup $H$ of $G$ and some linear character $\lambda \in \operatorname{Irr}(H)$ such that $X=\lambda^{G}$. We say $X$ is induced by $\lambda$. In this paper we find a way of locating proper subgroups of $G$ which have a character that induces $X$.

The notation in this paper follows that used in Isaacs [1]. The symbol $\phi(G)$ will denote the Frattini subgroup of $G$, the intersection of all maximal subgroups of $G$. For $X$ a character of $G, V(X)=\langle g \in G: X(g) \neq 0\rangle$ is called the support group of $X$ and is the smallest subgroup of $G$ outside of which $X$ vanishes. If $N$ is a normal subgroup of $G$ and $\psi \in \operatorname{Irr}(N)$, then $I_{G}(\psi)=\left[g \in G: \psi^{g}=\psi\right)$ is the inertia group or $\psi$ in $G$. If $\psi$ is an irreducible constituent of $X_{N}$ then we know there is some $\theta \in \operatorname{Irr}\left(I_{G}(\psi)\right)$ such that $\theta^{G}=X$. The main result of this paper is the following:

THEOREM 1.1: Let $G$ be a finite $p$-group and let $\chi$ be a non-linear irreducible character of $G$. Let $N$ be a normal subgroup of $G$ such that $V(X) \leq N \leq V(X) \phi(G)$ and let $\Psi$ be an irreducible constituent of $X_{N}$. If $\psi$ is non-linear then $I_{G}(\psi)<G$. 
This theorem enables us, by induction on the order of $G$, to form chains of subgroups with associated characters. Each of these characters induces $X$.

\section{PRELIMINARIES}

Besides Clifford's Theorem, Frobenius Reciprocity and the other fundamentals of character theory we will need the following results. The first is a corollary to a theorem of Isaacs[2]:

PROPOSITION 2.1 : Let $N$ be a normal subgroup of $G,|G: N|=p, p$ a prime. Suppose $X \in \operatorname{Irr}(G)$. Then either

a) $X_{N} \in \operatorname{Irr}(N)$

p

or b) $X_{N}=\sum_{i=1} \theta_{i}$ where $\theta_{1}$ are distinct irreducible characters of $N$

Let $\theta \in \operatorname{Irr}(N)$. Then either

D

a) $\theta^{G}=\sum X_{1}$ where $X_{1}$ are distinct irreducible characters of $G$ $i=1$

or b) $\theta^{G} \in \operatorname{Irr}(G)$

Futhermore, if $\phi$ is an irreducible constituent of $X_{N}$ and $X$ satisfies a (respectively $b$ ) of the first part then $\phi$ satisfies a (respectively $b$ ) of the second part. If $\psi$ is an irreducible constituent of $\theta^{G}$ and $\theta$ satisfies a (respectively $b$ ) of the second part then $\psi$ satisfies a (respectively $b$ ) of the first part.

LEMMA 2.2: Let $X$ be a non-linear irreducible character of $G$. Let $N$ be a normal subgroup of $G$ with $|G: N|=p, p$ a prime, and $N \geq V(X)$. If $\Psi$ is an irreducible p

constituent of $\chi_{N}$, then $\psi^{G}=X$ and $\chi_{N}=\sum \psi_{i}$, where $\psi_{i} \in I r r(N)$ are distinct. $i=1$

PROOF: The fact that $\psi$ is a constituent of $X_{N}$ implies that $X$ is a constituent of $\psi^{G}$ by Frobenius Reciprocity. Suppose $\theta \in \operatorname{Irr}(G)$ such that $\theta$ is a constituent of $\psi^{G}$. Then $\psi$ is also a constituent of $\theta_{N}$, thus $\left[X_{N}, \theta_{N}\right]=0$. Since $N \geq V(X), X$ vanishes outside of $N$. Thus, by definition of inner product, we have

$$
|G|[X, \theta]=\sum_{g \in G} X(g) \theta\left(g^{-1}\right)=\sum_{g \in N} X(g) \theta\left(g^{-1}\right)=|N|\left[\chi_{N}, \theta_{N}\right] .
$$


Hence $[X, \theta] \neq 0$ yellding $X=\theta$. By lemma $(2.1)(b)$ we have $\psi^{G}=X$ and $X_{N}=\sum_{i=1} \psi_{i} . / /$

PROPOSITION 2.3: Let $G$ be a $p$-group with a non-linear irreducible character $X$. Let $\theta$ be an irreducible constituent of $X V(X)$. If $\theta(1)=1$, then $I_{G}(\theta)<G$.

PROOF: Assume $\theta(1)=1$ satisfies the above hypotheses. Now $\theta=\lambda^{V(X)}$ where $\lambda$ is a linear character of some subgroup $H$ of $V(X)$. Let $M$ be a maximal subgroup of $V(X)$ containing $H$. Then $\theta=(\lambda M) V(X)$ by transitivity of character induction. Since $M$ is normal in $V(X), \theta$ vanishes off of $M$. Thus $V(X)>M \geq V(\theta)$. Suppose $I_{G}(\theta)=G$. By Clifford's Theorem, we have $X V(X)=e \theta$. It follows that $X$ vanishes off of $V(\theta)$ which is properly contained in $V(X)$ by our above observation. This is impossible by the minimality of $V(X)$. Therefore $I_{G}(\theta)<G$. //

The proof of the following may be found in Isaacs [1,pg 82].

THEOREM 2.4: Let $N$ be a normal subgroup of $G, \theta \in \operatorname{Irr}(N)$ and $I=I_{G}(\theta)$. Let $A=\left\{\psi \in \operatorname{Irr}(1):\left[\psi_{N}, \theta\right] \neq 0\right\}, B=\left\{X \in \operatorname{Irr}(G):\left[X_{N}, \theta\right] \neq 0\right\}$. Then

i) If $\psi \in A$ then $\psi \rightarrow \psi^{G}$ is a bijection of $A$ onto $B$

ii) If $\psi^{G}=X$ with $\psi \in A$ then $\psi$ is the unique irreducible constituent of $X_{1}$ which

lies in $A$ and $\left[\Psi_{N}, \theta\right]=\left[\chi_{N}, \theta\right]$.

\section{PROOF OF THEOREM 1.1}

Let $G$ be a $p$-group, $X \in \operatorname{Irr}(G), X(1)=1$, with $N$ a normal subgroup of $G$ such that $V(X) \leq N \leq V(X) \phi(G)$. Let $\psi$ be an irreducible constituent of $X_{N}$. Assume $I_{G}(\psi)=G$. We want to show that $\psi(1)=1$.

If $X$ is not falthful, replace $G$ by $G /$ ker $X$. We may do this since every character of G/kerX is also a character of $G$. Now, we prove that every irreducible constituent of $X_{V(X)}$ is linear. Let $\theta \leq X_{V(X)}$ be irreducible. Assume $I_{G}(\theta)<G$. Let $M$ be a maximal subgroup of $G$ such that $M \geq I_{G}(\theta)$. Since $I_{G}(\theta) \geq V(X)$ and $M$ is maximal, it follows that $M \geq V(X) \phi(G) \geq N$. By Lemma 2.2 we have

$$
X_{M}=\sum_{i=1}^{p} \beta_{i}, \text { where } \beta_{i} \in \operatorname{Irr}(M) \text { are distinct. }
$$


Let $G=M k g$, so $\beta_{j}=\beta_{1} g^{j-1}$ by Clifford's Theorem. Now

$$
X V(X)=\left(X_{M}\right) V(X)=\sum_{i=1}^{p}\left(\beta_{i}\right) V(X)=e \sum_{X \in\left[G: I_{G}(\theta)\right]} \theta^{x}
$$

by (3.1) and Clifford's Theorem.

Also

$$
{ }^{\left(\beta_{1}\right)_{V(X)}}=\uparrow \sum_{m \in\left[M \cdot I_{G}(\theta)\right]} \theta^{m} .
$$

Thus

$$
\left(\beta_{k}\right) V(X)=f \sum_{m \in\left[M I_{G}(\theta)\right]} \theta^{m g^{k-1}} .
$$

Clearly, $(m)$ being a transversal for $\left[M I_{G}(\theta)\right]$ implies that $\left(m g^{k-1}\right]$ is a transversal for $\left[G: I_{G}(\theta)\right]$. Since, by (3.2) and (3.4),

$$
\sum_{X \in\left[G: I_{G}(\theta)\right]}=\sum_{i=1}^{p}\left(\beta_{i}\right) V(X)=\sum_{i=1}^{p} \sum_{m \in\left[M: I_{G}(\theta)\right]} \theta^{i-1}
$$

we obtain $f=e$ and $\left(\beta_{1}\right)_{V}(X)$ and $\left(\beta_{j}\right) \vee(X)$ have no common constituents for $i_{j}$. But $I_{G}(\psi)=G$ so by Clifford's Theorem $X_{N}=a \psi$, yielding

$$
a \psi=X_{N}=\left(X_{M}\right)_{N}=\sum_{i=1}^{p}\left(\beta_{i}\right)_{N} .
$$

Thus $\left(\beta_{i}\right)_{V(X)}=(a / p) \psi$ all $i=1 \ldots p$ and so $\left(\beta_{1}\right)_{V(X)}=\left(\left(\beta_{1}\right)_{N}\right)_{V(X)}=(a / p) \psi V(X)$

for all $i$. This is impossible since the characters $\left(\beta_{1}\right)_{V(X)}$ have no common constituents. So $I_{G}(\theta)=G$ and, by Proposition $2.3, \theta$ is linear. Now show that $V(X)=N$. Again let $\theta \in \operatorname{Irr}(V(X))$ such that $\theta \leq X V(X)$. By the above argument $\theta$ is linear and it follows that $X V(X)=e \theta$ so $Z(X) \geq V(X)$, where $Z(X)$ denotes the center of $X$. Thus $Z(X)=V(X)$ as $Z(X)$ is always contained in $V(X)$. Suppose $V(X)<N$. Because $G$ is a $p$-group we can find $B$ normal in $G$ such that $V(X)<B \leq N$ and $|B: V(X)|=p$. Thus $V(X)=Z(G)$, since $Z(X)=Z(G)$. So $B$ is a cyclic extension of the center of $G$ and hence $B$ is abelian and all of its irreducible characters are linear. Now

$$
x_{B}=\uparrow \sum_{x \in\left[G: I_{G}(a)\right]}
$$

for some $a \in \operatorname{Irr}(B)$ where $f=\left[a, x_{B}\right]$. Since $a^{x}(b)=a\left(x b x^{-1}\right)=a(b)$ for all $b \in B$ and 
$x \in C_{G}(B)$ we obtain $C_{G}(B) \leq I_{G}(a)$. suppose $C_{G}(B)<I_{G}(a)$. Then by maximality of $C_{G}(B)$, $I_{G}(\alpha)=G$. This would mean that $\chi_{B}=f \alpha$ and $B \leq Z(X)$, an obvious contradiction. Thus $I_{G}(\alpha)=C_{G}(B)$ and $I_{G}(\alpha)$ is maximal in $G$ so

D

$\chi_{B}=f \sum_{i=1} \alpha_{i}$, where $\alpha_{i}$ are distinct irreducible linear characters, $\alpha=\alpha_{1}$.

Therefore $I_{G}(\alpha)$ is a maximal subgroup containing $B \geq V(X)$. Thus $I_{G}(\alpha) \geq V(X) \phi(G) \geq N$. Now since $I_{G}(\psi)=G$ we have $X_{N}=e \psi$. Hence

$$
f \sum_{i=1}^{D} \alpha_{1}=\chi_{B}=\left(\chi_{N}\right)_{B}=e \psi_{B} \text {. }
$$

It follows that

$$
\Psi_{B}=(f / e) \sum_{j=1}^{p} \alpha_{f} ;
$$

thus $\alpha$ is not invariant in $\mathrm{N} \mathrm{so}_{G}(\alpha)$ does not contain $\mathrm{N}$. This is a contradiction, so $V(X)=N$. Since all constituents of $X_{V(X)}=X_{N}$ are linear we have $\psi(1)=1$ as required.//

\section{CHARACTERS THAT INDUCE $X$}

In Theorem 1.1 we considered certain subgroups of $G$. Now we will examine the relationship of some characters associated with these subgroups.

PROPOSITION 4.1: Let $\chi$ be a non-linear irreducible character of $G$. Let $N$ be a normal subgroup of $G$ with $N 2 V(\chi)$. Suppose $\theta$ is an irreducible constituent of $\chi_{N}$, then $\theta^{G}=e \chi$ where $e^{2}=\left|I_{G}(\theta): N\right|$.

PROOF: Since $\theta$ is a constituent of $X_{N}$ we have $\left[\theta, X_{N}\right]=0$. By Frobenius Reciprocity, $\left[X, \theta^{G}\right]=0$ thus $X$ is a constituent of $\theta^{G}$. Suppoose $\psi \in \operatorname{Irr}(G)$ is a constituent of $\theta^{G}$. Then $0=\left[\theta^{G}, \psi\right]=\left[\theta, \Psi_{N}\right]$, so $\left[X_{N}, \psi_{N}\right]=0$ since $\theta$ is a constituent of both $\chi_{N}$ and $\Psi_{N}$. Since $N \geq V(X), X$ vanishes outside of $N$. Hence, by definition of inner product

$$
|G|[X, \psi]=\sum_{g \in G} X(g) \psi\left(g^{-1}\right)=\sum_{g \in N} X(g) \psi\left(g^{-1}\right)=|N|\left[X_{N}, \Psi_{N}\right]
$$


Thus $[X, \psi]=0$ and so $X=\psi$ since they are both irreducible. It follows that $X$ is the unique irreducible constituent of $\theta^{G}$, so $\theta^{G}=e X$. By definition of induced character $\theta^{G}(1)=|G: N| \theta(1)$, so $|G: N| \theta(1)=\operatorname{ex}(1)$. By Frobenius Reciprocity, $e=\left[\theta^{G}, X\right]=\left[\theta, X_{N}\right]$. Clifford's Theorem gives

$$
\begin{aligned}
& X(1)=X_{N}(1)=\text { e } \sum \theta^{x}(1)=\text { el } G: I_{G}(\theta) \mid \theta(1) \\
& x \in\left[G: I_{G}(\theta)\right]
\end{aligned}
$$

Thus

$$
\text { IG: } N \mid \theta(1)=e\left(e\left|G: I_{G}(\theta)\right| \theta(1)\right)
$$

It follows that $\mathrm{e}^{2}=\left|\mathrm{I}_{\mathrm{G}}(\theta): \mathrm{N}\right| . / /$

PROPOSITION 4.2: Let $G$ be a $p$-group with a non-linear irreducible character $X$. Let $N$ be a normal subgroup of $G$ such that $N \geq V(X)$ and let $\psi$ be an irreducible constituent of $X_{N}$. Let $1=I_{G}(\psi)$ and let $\beta$ be an irreducible constituent of $\psi$ '. Then $\psi^{\prime}=e \beta, e^{2}=|1: N|$ and $\beta^{G}=\chi$.

PROOF: By Proposition 4.1, $\psi^{G}=e x$ where $e^{2}=|1: N|$. We have $0=\left[\beta, \psi^{\prime}\right]=\left[\beta_{N}, \psi\right]$ by Frobenius Reciprocity. Now 2.4 tells us that $\beta^{G}$ is irreducible. Also $\beta^{G} s\left(\psi^{\prime}\right)^{G}=$ eX since $\beta$ is a constituent of $\psi^{\prime}$, so that $\beta^{G}=\chi$. Again by Proposition 2.4 , we have $\left[\beta_{N}, \psi\right]=\left[X_{N}, \psi\right]=$ e. Thus $e=\left[\beta, \psi^{\prime}\right]$ by Frobenius Reciprocity and it follows that $\psi^{\prime} \geq$ e $\beta$ since $\beta$ is irreducible. By definition of induced character, $\psi^{\prime}=|1: N| \psi(1)=$ $e^{2} \psi(1)$, so $e^{2} \geq e \beta(1)$. Since $\beta_{N} \geq$ e $\psi$ we have $\beta(1) \geq e \psi(1)$. Thus $e^{2} \psi(1)=\psi^{\prime}(1) \geq e \beta(1)$ $2 e(e \psi(1))$. It follows that $\psi^{\prime}(1)=e \beta(1)$ so $\psi^{\prime}=e \beta$. // Now for $X \in \operatorname{Irr}(G)$, we define an inertial decomposition series for $X$. denoted $\left[I_{i}, N_{i}, \beta_{i}, \Psi_{i}\right]_{i=0}^{m}$. Here $I_{0}=G=N_{0}, \beta_{0}=X=\Psi_{0}, N_{i}$ is normal in $I_{i}$, $I_{j+1}=I_{l_{i}}\left(\psi_{i+1}\right)$ for some $\psi_{i=1} \in \operatorname{Irr}\left(N_{i+1}\right), \beta_{i} \in \operatorname{Irr}\left(I_{i}\right)$ and $\left(\beta_{i+1}\right)^{l_{i}}=\beta_{1}$. Hence we have a chain of subgroups

$$
I_{m} s I_{m-1} s \ldots s I_{1} s I_{0}=G
$$

with associated characters $\beta_{i} \in \operatorname{Irr}\left(I_{i}\right)$ such that $\beta_{i}{ }^{G}=X$, all $i=1 \ldots m$, by transitivity of character induction. 
PROPOSITION 4.3: Let $G$ be a $p$-group with $\chi \in I r r(G)$. Then $\chi$ has an inertial $\mathrm{m}$

decompositon series $\left[l_{i}, N_{i}, \beta_{i}, \psi_{i}\right]_{i=0}$ with $\left(\psi_{i}\right)^{l_{i}}=e_{i} \beta_{i}$ where $e_{i}^{2}=\mid l_{i}$ : $N_{i} \mid$ and $V\left(\beta_{1}\right) \leq N_{1+1} \leq V\left(\beta_{1}\right) \phi\left(l_{1}\right),\left(\beta_{1}\right)_{N_{1}}=e_{1} \psi_{i}$ and $\Psi_{m}(1)=1, \Psi_{i} \neq 1$ for $1=1 \ldots m-1$. Furthermore, $\beta_{1}{ }^{G}=X$ for $i=1 . . m$.

PROOF: If $X$ is linear then it has a trivial inertial decomposition series, $\left[I_{0}, N_{0}, \beta_{0}, \Psi_{0}\right]$. Assume $X$ is non-linear. Proof is by induction on $|G|$. Let $N$ be a normal subgroup of $G$ satisfying $V(X) \leq N \leq V(X) \phi(G)$. Let $\Psi$ be an irreducible constituent of $X_{N}$

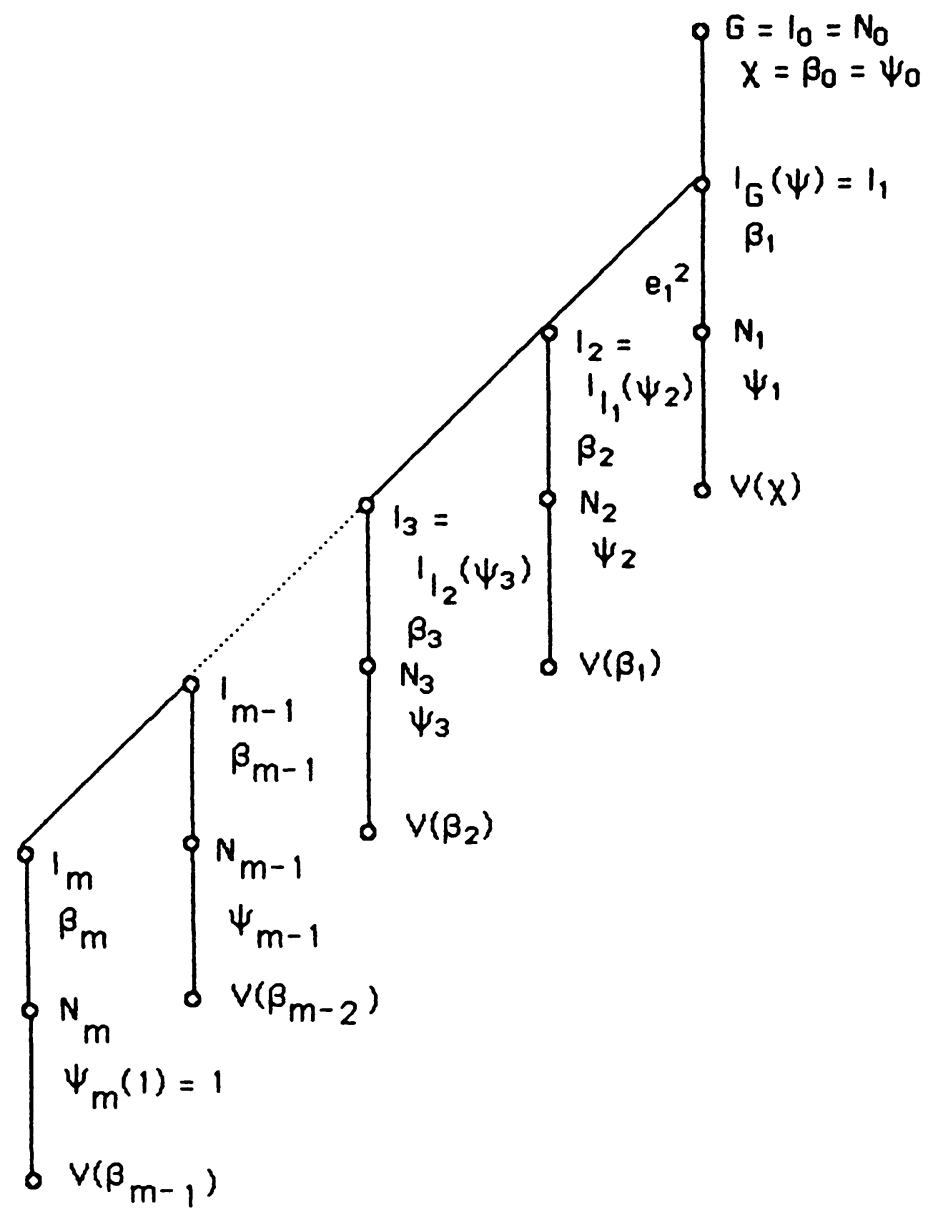

Figure 1

and let $1=I_{G}(\psi)$. Let $\beta$ be a irreducible constituent of $\psi^{\prime}$. By Proposition $4.2, \beta^{G}=\chi$, $\psi^{i}=e \beta$ where $e^{2}=|I: N|$, and $\beta_{N}=e \psi$ by Clifford's Theorem. Set $I_{0}=G=N_{0}$, 
$\beta_{0}=\chi=\psi_{0}, N_{1}=N, I_{1}=1, \beta_{1}=\beta$, and $\psi_{1}=1$, then $\left[l_{i}, N_{i}, \beta_{1}, \psi_{i}\right]_{i=0}^{\prime}$ is an inertial decomposition series for $X$ as required.

Suppose $\psi(1)>1$. Then by Theorem 1.1, $<$. Also $\beta_{N}=e \psi$ implies that $\beta(1)=$ $e \psi(1)>1$. Since $\beta(1)>1$ we can apply our Induction hypothesis.//

Note that, in general, we do not have $l_{j+1}$ normal in $l_{i}$ nor $N_{j+1} \leq N_{j}$ in an inertial decomposition series. This inertial decomposition series is illustrated in Figure 1.

ACKNOWLEDEMENTS. Most of the results of this paper were contained in the author's doctoral dissertation [3], prepared under the supervision of K. M. Kronstein at the University of Notre Dame. Special thanks go to A. H. Clifford for helpful comments during the writing of this paper.

\section{REFERENCES}

1. ISAACS, I. M. :Characters of Finite Groups . Academic Press 1976.

2. ISAACS, I. M. :Fixed points and characters in groups with non-coprime operator groups. Canadian J. Math. 20, 1315-1320 (1968).

3. SANTA COLOMA, T. L.Monomial Characters Some Important Subgroup Series. Thesis, University of Notre Dame 1978. 


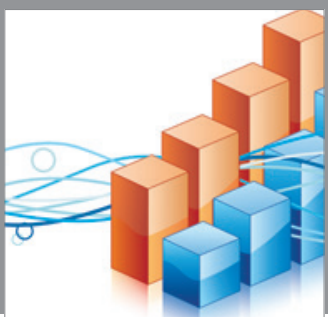

Advances in

Operations Research

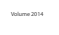

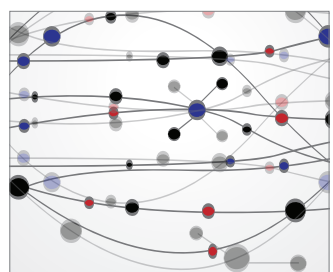

\section{The Scientific} World Journal
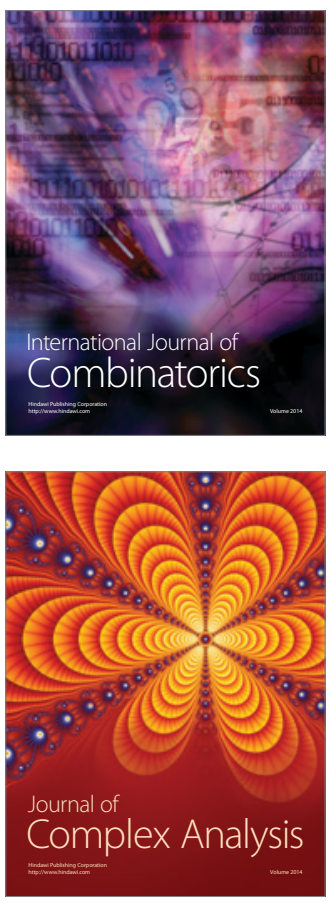

International Journal of

Mathematics and

Mathematical

Sciences
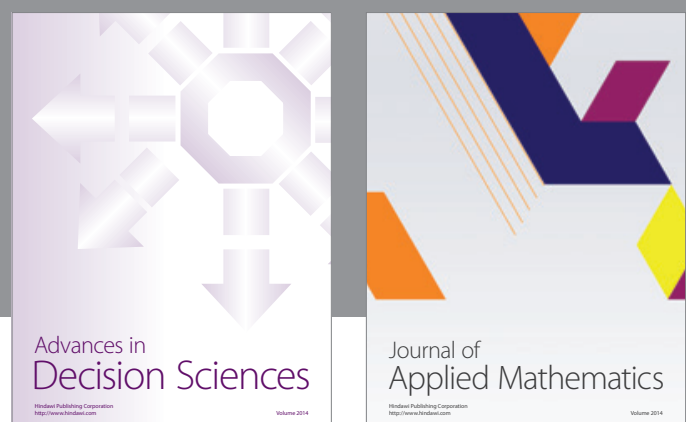

Journal of

Applied Mathematics
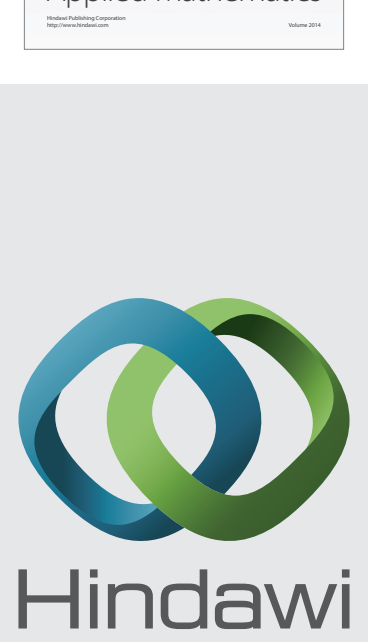

Submit your manuscripts at http://www.hindawi.com
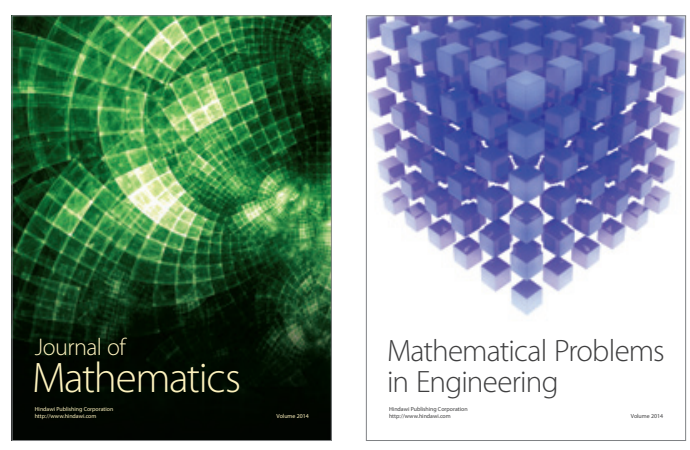

Mathematical Problems in Engineering
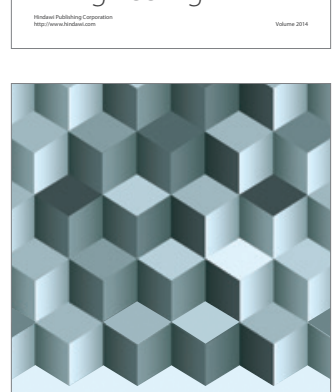

Journal of

Function Spaces
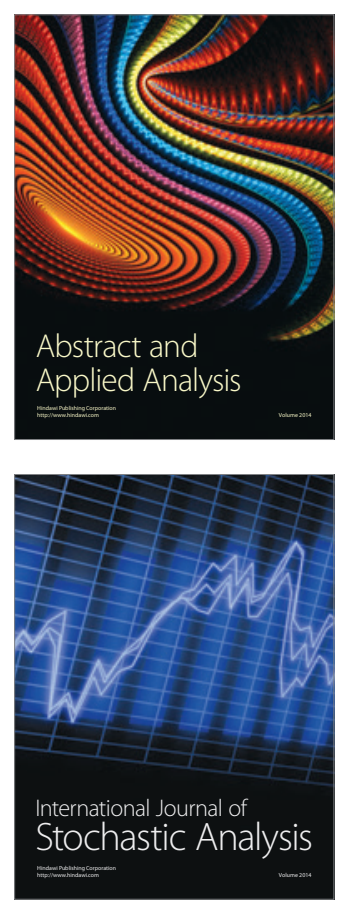

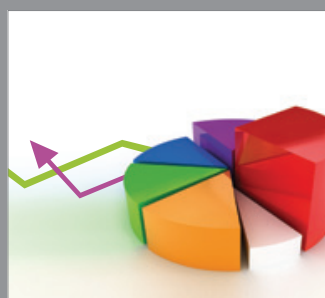

ournal of

Probability and Statistics

Promensencen
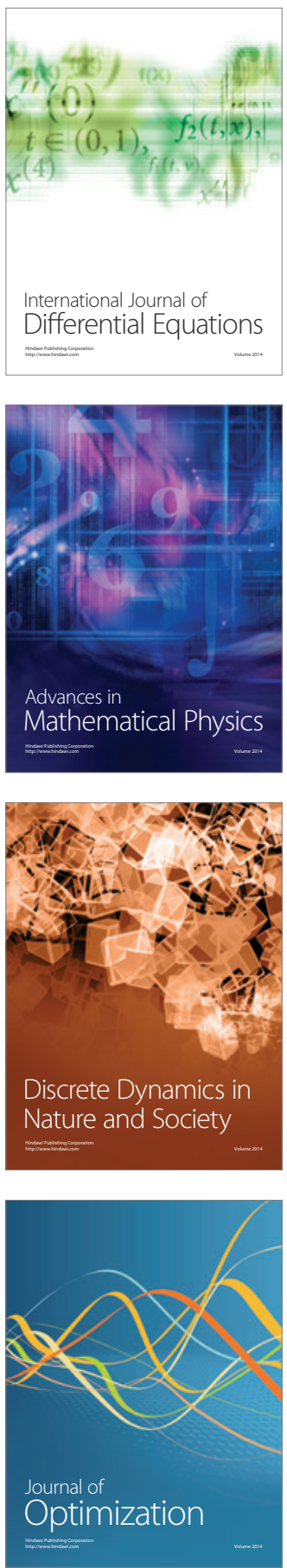